Reprod. Nutr. Dévelop., 1982, 22 (3), 439-453.

\title{
L'établissement de la puberté chez la lapine (Folliculogenèse et ovulation). Effet du rationnement alimentaire
}

\author{
Françoise HULOT, J. C. MARIANA $(*)$, F. LEBAS $(* *)$
}

Station d'Amélioration génétique des Animaux, I.N.R.A. B.P. 12, 31320 Castanet Tolosan.

(*) Station de Physiologie de la Reproduction, I.N.R.A. Nouzilly, 37380 Monnaie.

(**) Laboratoire de Recherches sur l'Elevage du Lapin, I.N.R.A. B.P. 12, 31320 Castanet Tolosan.

Summary. Establishment of puberty (folliculogenesis and ovulation) in the doe-rabbit. Effect of rationing the diet.

We used 307 Californian doe-rabbits divided into 7 experimental groups. The first 4 groups, fed an ad libitum diet, were presented to the male at 11, 14, 17 or 20 weeks of age. The other 3 groups were fed a 75 p. 100 ad libitum diet and were presented to the male at 14, 17 and 20 weeks of age. Three to 5 does per group were killed at 8 postcoitum hours to check the ovarian follicles, and the remaining females, after effective mating, were killed 12 days later to check the ovulation rate.

Acceptation of mating was homogeneous (75-80 p. 100) in all the groups, except in the one with the restricted diet presented to the male at 14 weeks of age (55 p. 100). In ad libitum-fed does, the percentage of females mated and ovulating reached one-third at 14 weeks and two-thirds at 17 and 20 weeks. Rationing retarded by 3 weeks live-weight gain and ability to ovulate. Whatever the diet given, the mean weight of does of this breed had to reach $3 \mathrm{~kg}$ before the first ovulation occurred and $3.3 \mathrm{~kg}$ before the percentage of females ovulating after mating became normal. The number of ova found in ovulating females did not differ statistically in any of the groups, but the rationed does tended to have fewer ova $(-8$ p. 100) in direct relation to their live weight. In fact, whatever the diet, the number of ova increased more than 2-units for a weight increase of $1 \mathrm{~kg}$ of live weight.

In ad-libitum-fed does, the follicle population doubled between 11 and 14 weeks, then decreased to attain at 20 weeks the value found at 11 weeks. Although this population was unimodal at 11 weeks, it became bimodal afterwards and was clearly so at 1720 weeks. The second mode represented 5 then 8 p. 100 of the total follicles which were large-sized (> $407000 \mu 2)$ and corresponded to preovulatory follicles. The number of atretic follicles followed a similar pattern. Rationing did not change the follicular population of the first mode ; on the contrary, it altered the size and number of the largesized follicle population of the second mode. Rationing also increased the percentage of atretic preovulatory follicles in the two oldest groups.

\section{Introduction.}

Le but de ce travail est de préciser au moment de la puberté l'évolution d'un certain nombre de facteurs (réceptivité sexuelle, poids corporel, population de 
follicules ovariens), conditionnant l'apparition des premières ovulations chez la lapine. L'expérience est menée de 11 semaines d'âge, peu après le stade "critique " de l'ovaire, marqué par l'apparition des follicules à antrum (Adams, 1954 ; Mauléon, 1961 ; Levasseur, 1979), jusqu'à 20 semaines, âge normal de mise en reproduction. Seule la population des follicules à antrum a fait l'objet d'une étude quantitative et qualitative minutieuse. Ce travail est conduit à la fois sur des lapines alimentées à volonté et d'autres recevant une quantité restreinte d'aliment de manière à observer l'impact éventuel d'un retard de croissance sur la réceptivité sexuelle, la folliculogenèse et l'ovulation. On sait en effet qu'en règle générale, chez les autres espèces, la restriction alimentaire entraîne un retard dans le démarrage de la puberté (Widdowson et al., 1964 ; Lintern-Moore et Everitt, 1978).

\section{Matériel et méthodes.}

320 femelles de race californienne mises en cage individuelle à l'âge de 8 semaines (poids moyen $1750 \pm 150 \mathrm{~g}$ ) sont alimentées à volonté avec un aliment standard commercial jusqu'à 11 semaines, date à laquelle un rationnement limité à $150 \mathrm{~g} /$ jour est effectué sur la moitié du cheptel ; l'autre moitié est toujours nourrie ad libitum, ce qui correspond à une consommation moyenne d'environ $200 \mathrm{~g} /$ jour. La répartition des femelles entre ces deux types d'alimentation a tenu compte de leur parenté (pleines sœurs et demi-sœurs réparties également dans les 2 lots) et de leurs caractéristiques pondérales de manière à assurer au départ le même poids moyen dans chaque série.

Les examens effectués sont réalisés à 11 semaines (1 régime), 14, 17 et 20 semaines (2 régimes). La planification de l'expérience a été conçue de telle manière que les animaux appartenant aux quatre groupes d'âge soient contrôlés simultanément. Ce plan a été réalisé sur une période de 3 semaines consécutives (28 septembre, 5 octobre et 12 octobre) pour éviter toute interaction entre l'âge des femelles et la saison.

Les 7 lots d'animaux ainsi constitués comprenaient a priori 40 lapines chacun, à l'exception du premier (11 semaines) d'un effectif double. Les contrôles suivants ont été réalisés :

- Poids vif : chaque femelle est pesée d'une part aux âges standards (11 - 14 17 - 20 semaines) d'autre part au moment de la saillie (qui intervient 2 à 4 jours plus tard) ou lors de son élimination pour refus d'accouplement.

- Réceptivité sexuelle : les lapines sont présentées à un premier mâle, puis en cas de refus, à un second un quart d'heure plus tard. Si la femelle n'est pas saillie le premier jour, les tentatives sont renouvelées les 2 jours suivants, limite au-delà de laquelle l'élimination est systématique.

- Performances d'ovulation: pour chaque lot, les femelles ayant accepté librement l'accouplement sont abattues 12 jours post coïtum afin de contrôler le nombre d'animaux ovulant et le nombre d'ovules pondus (dénombrement des corps jaunes). 
- Nombre et qualité des follicules ovariens à antrum : pour chaque groupe d'âge et régime alimentaire, 3 à 5 lapines saillies dès la première présentation sont sacrifiées 8 heures après le coït. Ce stade permet de déceler avec certitude les follicules pré-ovulatoires, caractérisés par des ovocytes reprenant la méïose et expulsant le premier globule polaire (Cherney et al., 1975; Thibault et al., 1976). L'ovaire droit est prélevé, placé 3 jours dans du fixateur Bouin-Hollande. Après déshydratation et inclusion dans la cytoparaffine, des coupes sériées de $10 \mu$ sont réalisées et colorées à l'hématoxyline de Regaud.

La population étudiée est celle des follicules à antrum (surface égale ou supérieure à $26800 \mu_{2}$, ce qui correspond à un diamètre de $185 \mu$ ). Cette surface est mesurée à l'aide d'un analyseur d'images Leitz semi-automatique, au niveau de la coupe contenant le nucléole de l'ovocyte ou le premier globule polaire. Sur cette même section est estimée la qualité du follicule : ceux présentant plus de 10 cellules pycnotiques sont considérés comme atrésiques.

Pour analyser les données recueillies, les follicules ont été séparés en 14 classes de taille, fixées arbitrairement, dont les limites sont en progression géométrique de raison, 1,40 (Mariana, 1972). Les distributions ainsi obtenues aux différents âges et pour différents régimes ont été comparées par le test de Kolmogorov Smirnov (K.S.). L'influence de l'âge et du régime alimentaire sur le taux d'ovulation a été étudiée au moyen du test $\chi^{2}$ et celle sur le nombre d'ovules pondus par des analyses non paramétriques (Wilcoxon, Wilson).

\section{Résultats.}

1. L'évolution pondérale. - Le tableau 1 indique les moyennes de poids enregistrées d'une part sur la totalité des lapines présentes aux différents âges standards, d'autre part sur les femelles constituant chacun des lots.

Pour une alimentation et un âge donné, le lot de femelles destinées aux contrôles biologiques a un poids moyen ne différant pas significativement de l'ensemble des femelles pesées au même âge. Chaque lot est par conséquent un échantilion représentatif de la population générale.

La croissance des femelles alimentées à volonté est pratiquement linéaire entre 11 et 20 semaines, soit un gain de $21 \mathrm{~g} /$ jour en moyenne. Le rationnement réduit significativement le poids vif des femelles de 8 à 10 p. 100 (tabl. 1).

2. La réceptivité sexuelle. - Entre 11 et 20 semaines, environ 80 p. 100 des femelles nourries ad libitum, présentées au mâle, sont saillies (fig. 1). Chez les animaux rationnés, 3 semaines après le début de la restriction alimentaire, une réduction du taux d'acceptation du mâle se manifeste : 55 p. 100 des femelles sont saillies ; ce chiffre est significativement inférieur à ceux observés à 17 et 20 semaines (75 et 86 p. 100 respectivement), âges auxquels ces taux sont comparables aux valeurs du témoin (88 et 82 p. 100) alimenté à volonté.

Dans chaque lot, le poids moyen des femelles acceptant le mâle ne diffère pas de celui des femelles le refusant (tabl. 2). Cependant, dans le lot de lapines de 20 semaines nourries à volonté, la différence est significative (femelles non 
réceptives plus légères que les réceptives, mais les effectifs sont disproportionnés : 5 et 31 femelles respectivement).

\section{TABLEAU 1}

Evolution du poids moyen de l'ensemble des lapines aux différents âges standards, et poids moyen des lots expérimentaux (moyenne et écart-type de la population).

\begin{tabular}{|c|c|c|c|c|c|}
\hline \multirow{2}{*}{$\begin{array}{c}\text { Age } \\
\text { (semaines) }\end{array}$} & \multirow{2}{*}{$\begin{array}{l}\text { Groupes de } \\
\text { lapines }\end{array}$} & \multicolumn{2}{|c|}{ Femelles ad libitum } & \multicolumn{2}{|c|}{ Femelles rationnées } \\
\hline & & Nombre & $\begin{array}{l}\text { Poids (g) } \\
\bar{x} \pm s\end{array}$ & Nombre & $\begin{array}{l}\text { Poids }(g) \\
\bar{x} \pm s\end{array}$ \\
\hline \multirow{2}{*}{11} & $\begin{array}{l}\text { Totalité des } \\
\text { femelles } \ldots \ldots \ldots\end{array}$ & 307 & $2334 \pm 178$ & - & - \\
\hline & Lot expérimental & 76 & $2449 \pm 188$ & - & - \\
\hline \multirow{2}{*}{14} & $\begin{array}{l}\text { Totalité des } \\
\text { femelles ....... }\end{array}$ & 115 & $2923 \pm 223$ & 116 & $2679 \pm 149$ \\
\hline & Lot expérimental & 39 & $3018 \pm 228$ & 38 & $2740 \pm 148$ \\
\hline \multirow{2}{*}{17} & $\begin{array}{l}\text { Totalité des } \\
\text { femelles .... }\end{array}$ & 76 & $3383 \pm 267$ & 78 & $3029 \pm 187$ \\
\hline & Lot expérimental & 40 & $3454 \pm 257$ & 39 & $3076 \pm 200$ \\
\hline 20 & $\begin{array}{l}\text { Totalité des } \\
\text { femelles } \\
(=\text { lot expérim.) }\end{array}$ & 36 & $3655 \pm 347$ & 39 & $3339 \pm 202$ \\
\hline
\end{tabular}

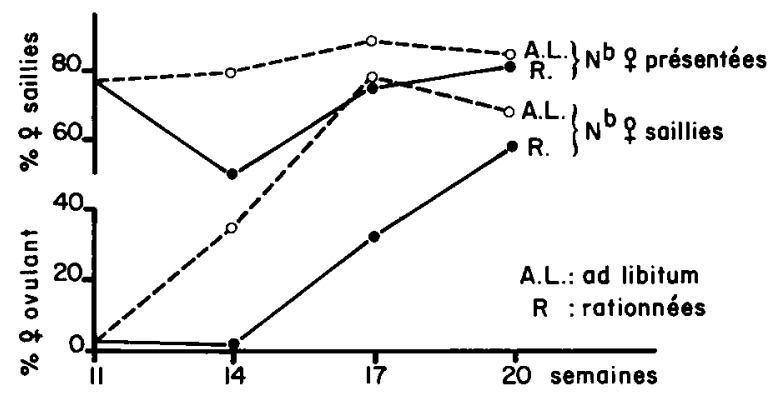

FIG. 1. - Evolution du pourcentage de lapines saillies et de lapines ayant ovule en fonction de l'âge. Effet du rationnement.

On peut donc affirmer que le rationnement alimentaire a un effet transitoire sur le taux de femelles qui acceptent de s'accoupler dans nos conditions standardisées, mais que cet effet est indépendant du poids vif individuel des lapines. 


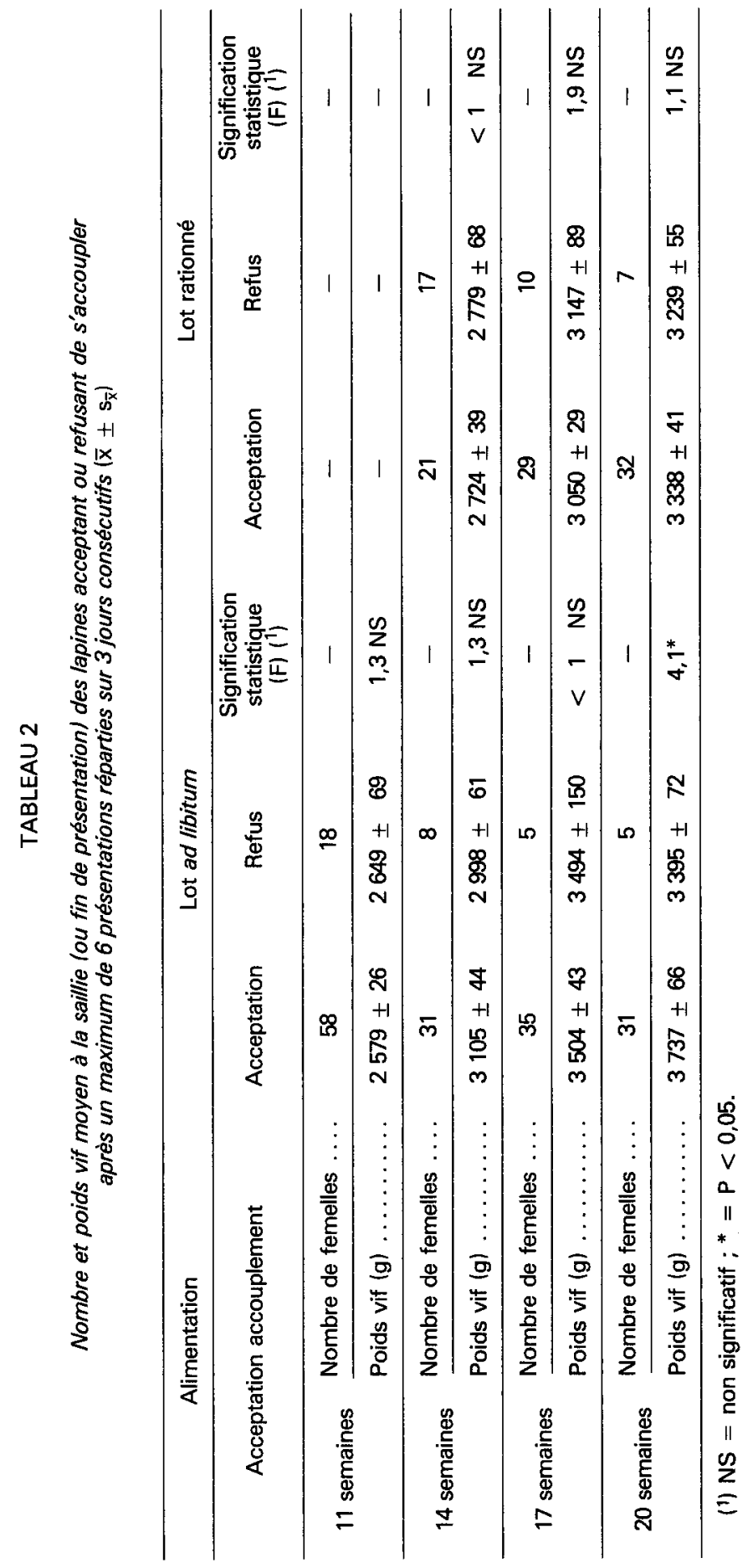




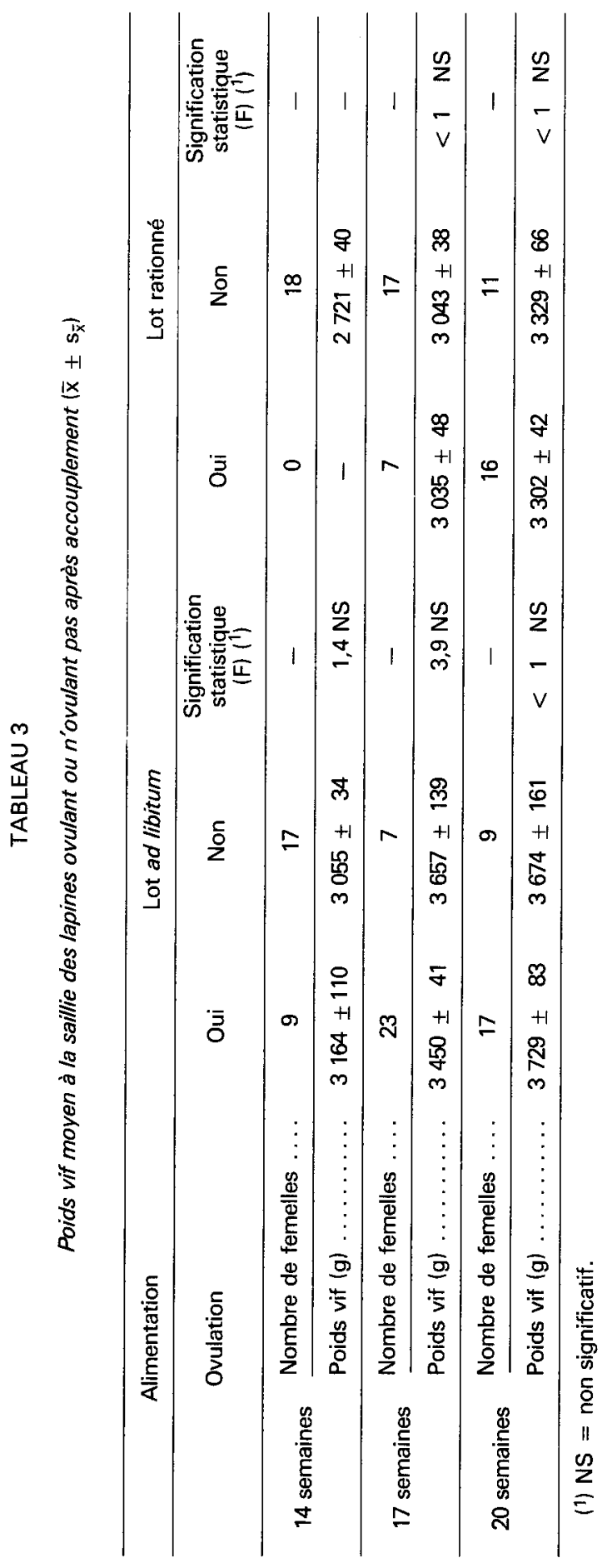




\section{L'ovulation.}

3.1. L'aptitude des femelles à ovuler. - Les observations portent sur les femelles abattues 12 jours après la saillie, soit 205 animaux.

- Une femelle nourrie ad libitum sur 54 ovule à 11 semaines, le 1/3 environ à 14 semaines et les $2 / 3$ à l'âge de 17 semaines. Le rationnement retarde d'environ 3 semaines cette évolution. Le lot de femelles rationnées acquiert seulement à 20 semaines une aptitude à ovuler comparable à celle atteinte par les témoins dès 17 semaines (fig. 1 ).

- Quel que soit le régime alimentaire, le poids moyen des lapines de cette souche doit atteindre $3 \mathrm{~kg}$ pour que certaines femelles du groupe ovulent. Aux environs de $3300-3400 \mathrm{~g}$, le pourcentage de femelles ovulant tend à se stabiliser (tabl. 3). Aucune différence significative n'apparaît entre le poids moyen à la saillie des femelles ovulant ou n'ovulant pas, à l'exception du lot de femelles âgées de 17 semaines nourries ad libitum (différence significative à 10 p. 100).

3.2. Le nombre d'ovules pondus. - Une analyse de variance non paramétrique montre qu'il n'y a pas de différences significatives entre les moyennes des nombres d'ovulations aux différents âges à l'intérieur de chaque groupe d'alimentation (tabl. 4). Par contre, globalement, les animaux restreints ont une ponte ovulaire un peu inférieure et une variabilité moindre que les animaux nourris ad libitum (significatif à $5 \mathrm{p}$. 100). Cette réduction peut être rapportée au faible poids moyen des femelles restreintes qui ovulent, par rapport à celui des femelles alimentées à volonté. En effet, sur l'ensemble des lapines ovulant, nous observons une corrélation positive significative $(r=0,33)$ entre le nombre d'ovules pondus et le poids corrigé des femelles. La correction qui permet de différencier l'effet âge de l'effet poids consiste à ajouter au poids de chaque femelle la valeur algébrique de l'écart entre le poids moyen du lot lâge $x$ régime) auquel elle appartient et le poids moyen général de l'ensemble des femelles ovulant. Si on considère séparément les groupes ad libitum et rationnés, les coefficients de corrélation sont respectivement $r=0,32$ et $r=0,45$. Sur l'ensemble, l'équation de régression est $y=2,6 x-0,338$ où $y$ est le nombre d'ovules pondus et $x$ le poids vif à la saillie de la femelle exprimé en

TABLEAU 4

Nombre moyen d'ovules pondus par les femelles ayant ovule $\left(\bar{x} \pm \mathrm{s}_{\overline{\mathrm{x}}}\right)$

\begin{tabular}{cccc}
\hline Alimentation & Lot ad libitum & Lot rationnée & $\begin{array}{c}\text { Signification } \\
\left.\text { statistique ( }{ }^{(}\right)\end{array}$ \\
\hline 11 semaines $\ldots \ldots \ldots \ldots$ & $(10)$ & - & - \\
\hline 14 semaines $\ldots \ldots \ldots \ldots$ & $8,67 \pm 1,50$ & - & - \\
\hline 17 semaines $\ldots \ldots \ldots \ldots$ & $8,26 \pm 2,13$ & $7,88 \pm 0,99$ & NS \\
\hline 20 semaines $\ldots \ldots \ldots \ldots$ & $9,24 \pm 2,51$ & $8,00 \pm 1,30$ & $*$ \\
\hline
\end{tabular}

(1) NS = non significatif ; ${ }^{*} \mathrm{P}<0,05$. 


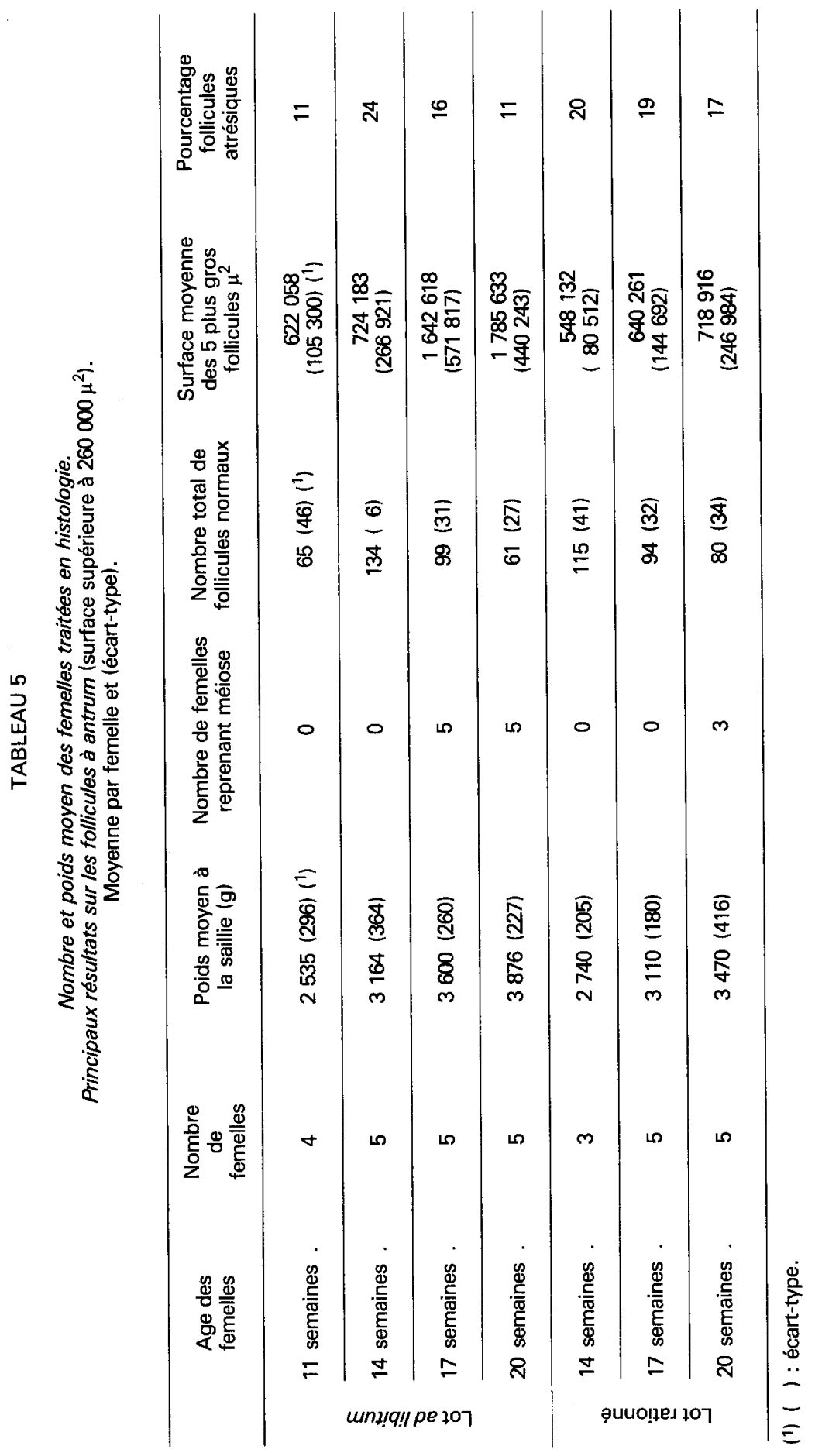


kilogrammes. Ainsi, dans un groupe donné de femelles, une première lapine pesant $1 \mathrm{~kg}$ de plus qu'une autre donnera a priori 2,6 ovules de plus que la seconde.

4. La croissance folliculaire. - Le tableau 5 résume les principaux résultats obtenus sur les 32 femelles abattues $8 \mathrm{~h}$ après la saillie. Le poids moyen de ces femelles dans chaque lot est comparable à celui de l'ensemble des femelles saillies (voir tabl. 2). Les critères d'études retenus sont le nombre, la surface et la reprise de l'activité nucléaire dans la population des follicules à antrum et atrésiques.

\subsection{Follicules normaux à antrum.}

- Le nombre moyen de follicules double de 11 à 14 semaines puis décroît chez les animaux nourris ad libitum. A 20 semaines, il est voisin du nombre compté à

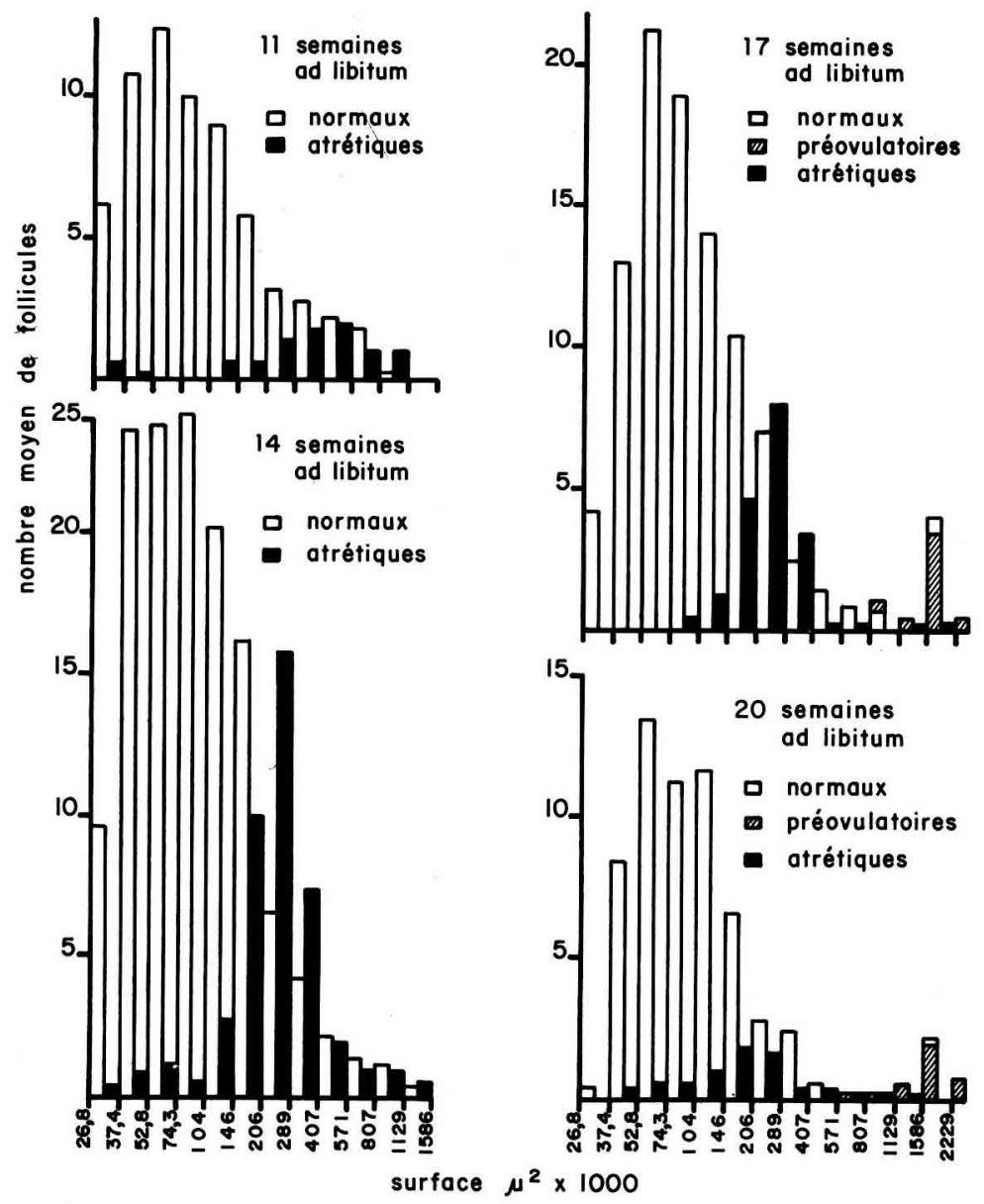

FIG. 2. - Lapines alimentées ad libitum. Histogramme des follicules à antrum normaux et atrésiques aux différents âges (moyenne par femelle). 
11 semaines (tabl. 5). II existe une régression significative $(r=-0,80 \pm 0,09$ ) du nombre total des follicules avec l'âge des animaux entre 14 et 20 semaines. Le rationnement ne modifie pas sensiblement cette évolution.

- La distribution de la taille est unimodale chez les animaux âgés de 11 semaines. Un second mode se dessine dès 14 semaines chez les animaux nourris ad libitum (fig. 2), il est net à 17 et 20 semaines. Le premier mode est celui de la population constituée par environ 95 p. 100 des follicules dénombrés (surface comprise entre $26800 \mu^{2}$ et $407000 \mu^{2}$ ). II présente, de ce fait, des caractéristiques sensiblement équivalentes à celles de la population totale. La médiane de la distribution est très voisine de $74300 \mu^{2}$ à 11, 14, 17 et 20 semaines, ce qui indique une stabilité du renouvellement de la population des follicules à antrum. Le rationnement alimentaire ne modifie pas la distribution des follicules (fig. 3) et le test de K.S ne permet pas de déceler de différences significatives entre les 2 distributions pour chacune des périodes considérées au niveau de 5 p. 100. Le deuxième mode est celui d'une population peu nombreuse de follicules de grande surface (plus de $407000 \mu^{2}$ ) représentant environ $4 p .100$ de la population des follicules mesurés à 14 semaines, 8 p. 100 à 17 et 20 semaines. Avec l'âge croissant des animaux, le mode tend à se déplacer vers les grandes surfaces (maximum $2229000 \mu^{2}$ ). La taille moyenne des plus gros follicules (tabl. 5) augmente significativement de 11 à 17 semaines, âge auquel la croissance maximale paraît atteinte. Le rationnement entraîne des modifications dans la distribution de ces grands follicules. Leur nombre n'augmente pratiquement pas avec l'âge $(5$ p. 100 de la population totale à 17 et 20 semaines). L'évolution du mode vers les grandes tailles est limitée à
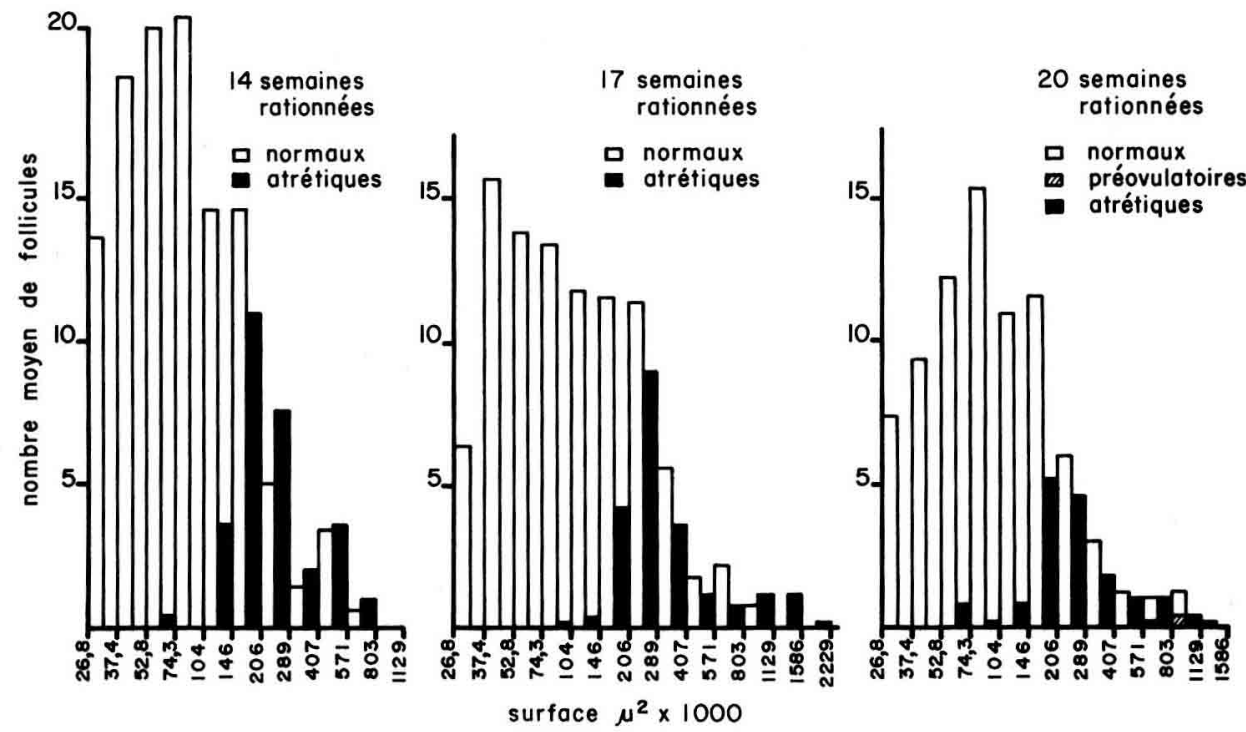

FIG. 3. - Lapines rationnées. Histogramme des follicules à antrum normaux et atrésiques aux différents âges (moyenne par femelle). 
$1129000 \mu^{2}$; la surface moyenne des plus gros follicules croît de 14 à 20 semaines, âge auquel elle reste très significativement inférieure à la valeur observée chez les animaux nourris à volonté.

La reprise de la méiose est observée chez toutes les femelles alimentées à volonté de 17 à 20 semaines et seulement chez trois femelles rationnées de 20 semaines sur les cinq étudiées. Chez ces animaux, la gamme de taille des follicules pré-ovulaires est très large pouvant aller de $571000 \mu^{2}$ à $2229000 \mu^{2}$ (figs. 2 et 3 ).

\subsection{Les follicules atrésiques.}

- Le nombre total de follicules atrésiques évolue au cours du temps comme celui des follicules normaux. Il passe par un maximum à 14 semaines et devient alors, chez les témoins, 5 fois plus élevé que l'effectif des follicules atrésiques observé à 11 semaines, et 3,4 fois plus important que ce nombre, chez les femelles rationnées.

Le taux d'atrésie, estimé par le rapport du nombre des follicules atrésiques sur le nombre total passe de 11 à 24 p. 100 entre 11 et 14 semaines chez les témoins (tabl. 5). II décroît également de façon significative entre 14 et 17 semaines, puis évolue peu entre 17 et 20 semaines. Chez les animaux rationnés, le taux d'atrésie est comparable à 14, 17 et 20 semaines. Par rapport aux animaux nourris à volonté, le rationnement entraîne une augmentation de l'atrésie perceptible à 17 semaines et significative à 20 semaines.

- Les classes de taille les plus touchées par la dégénérescence sont comprises entre $146000 \mu^{2}$ et $407000 \mu^{2}$ (figs. 2 et 3). La médiane de la distribution des follicules atrésiques est supérieure à celle de la distribution des follicules normaux quelque soit le régime alimentaire. Pour les tailles supérieures à $407000 \mu^{2}$, le nombre de follicules atrésiques semble décroître en fonction de la taille pour les femelles nourries ad libitum, ce qui est moins net pour les femelles rationnées.

Le taux d'atrésie des follicules de taille inférieure à $407000 \mu^{2}$ évolue peu chez les femelles nourries ad libitum et touche entre 8 et 23 p. 100 de la population (tabl. 6). Pour les follicules de taille supérieure à $407000 \mu^{2}$, il représente environ la moitié de la population à 11 et 14 semaines et décroît

TABLEAU 6

Pourcentage de follicules atrésiques dans les classes de petits et gros follicules

Follicules atrésiques (p. 100)

\begin{tabular}{ccccc}
\multirow{2}{*}{$\begin{array}{c}\text { Age } \\
\text { (semaines) }\end{array}$} & \multicolumn{2}{c}{ Entre 26800 et $\mathbf{4 0 7 0 0 0 \mu ^ { 2 }}$} & \multicolumn{2}{c}{ Supérieurs à $407000 \mu^{2}$} \\
\cline { 2 - 5 } & Lot ad libitum & Lot rationné & Lot ad libitum & Lot rationné \\
\hline 11 & 08 & - & 49 & - \\
\hline 14 & 23 & 18 & 47 & 53 \\
\hline 17 & 16 & 16 & 09 & 48 \\
\hline 20 & 10 & 15 & 18 & 40 \\
\hline
\end{tabular}


significativement à 17 et 20 semaines. Chez les animaux rationnés, le taux d'atrésie observé dans la population des follicules de taille inférieure à $407000 \mu^{2}$ ne diffère pas sensiblement de celui observé chez les témoins. Il est par contre supérieur dans la population des gros follicules et reste très élevé à 17 et 20 semaines. L'augmentation du taux d'atrésie consécutif au rationnement observé précédemment à 17 et 20 semaines est donc due essentiellement à l'accroissement de la dégénérescence des plus gros follicules à antrum.

\section{Discussion.}

1. La réceptivité sexuelle. - L'acceptation du mâle se manifeste très tôt, indépendamment du poids et de l'ovulation puisqu'à 11 semaines, le comportement sexuel n'est pas différent du comportement adulte. Asdell (1946) signalait que des cas de copulations peuvent survenir 2 mois avant la première ovulation. Yaschine et al. (1967) ont montré chez la lapine adulte que l'ovariectomie conduit à une baisse du comportement d'œstrus et que l'injection d'œstrogène le restaure. Par ailleurs, l'examen de l'ovaire à 11 semaines montre l'existence de follicules à antrum déjà bien développés et susceptibles d'excréter des stéroïdes. Si l'on admet que la "réceptivité " peut être en partie liée à la présence des stéroïdes ovariens, l'état de maturité de l'ovaire à 11 semaines serait donc suffisant pour permettre un comportement d'œestrus.

2. L'évolution pondérale et l'ovulation. - L'aptitude à ovuler survient plus tard (14 semaines) et de façon progressive. L'état de croissance de l'animal paraît une condition essentielle au démarrage de la ponte ovulaire : un poids moyen minimum d'environ $3 \mathrm{~kg}$ pour cette souche est nécessaire, soit les $3 / 4$ environ du poids adulte, ce qui est classiquement admis chez beaucoup de mammifères. Un gain de poids de 10 à 12 p. 100 permet d'obtenir un pourcentage "normal » de femelles ovulantes. Au-delà de $3,4 \mathrm{~kg}$, cette variable n'aurait plus d'influence sur le déclenchement de l'ovulation.

Le nombre d'ovules pondus est également conditionné par la croissance de l'animal ( $+2,6$ ovules par $\mathrm{kg}$ aux âges étudiés). II n'est pas exclu de penser que le poids continue à jouer un rôle au-delà de 20 semaines et contribue à l'accroissement du nombre d'ovules pondus observé chez des femelles plus âgées, primipares et multipares (Hulot et Matheron, 1981).

La relation poids-nombre d'ovules pondus avait déjà été notée chez le lapin par Gregory (1932) (1 ovule de plus pour un gain de poids de $434 \mathrm{~g}$ ) et par Venge (1950) (1 ovule pour $394 \mathrm{~g}$ ).

3. La croissance folliculaire et l'ovulation. - L'évolution de la population des follicules à antrum présente différentes caractéristiques :

- Les changements quantitatifs affectent en même temps les follicules normaux et atrésiques. Les variations du nombre de follicules sains ne résultent donc pas d'un processus d'atrésie régulateur. Elles sont probablement dues à des vitesses de croissances différentes, ce qui se traduit par un approvisionnement accru à certaines périodes de la vie et à un ralentissement à d'autres. Ces variations de vitesse de croissance seraient liées selon certains auteurs à l'âge de l'animal et à la taille des follicules (Pedersen, 1969, 1970, chez la souris). 
- A 11 et à 20 semaines, le nombre de follicules normaux et le pourcentage d'atrésie sont comparables après être passés par une phase d'accroissement spectaculaire à 14 semaines. Chez d'autres espèces animales, un certain nombre d'auteurs ont noté l'existence d'une augmentation du nombre de follicules normaux en croissance dans la période prépubertaire (souris: Peters, 1969, 21 jours ; rat : de Reviers, 1974, aux environs de 20 jours ; mouton : Kennedy et al., 1974, à 4 semaines), mais à notre connaissance aucune étude ne porte sur la seule population des follicules à antrum.

Ces fluctuations sont sans doute reliées à des variations des niveaux des hormones gonadotropes. On sait, par exemple, que chez la souris, une montée de FSH hypophysaire entre 16 et 20 jours (de Reviers, 1974) ou plasmatique à 12 et 15 jours selon les auteurs (Ojeda et Ramirez, 1972; Caligaris et al., 1973) précède l'apparition du nombre maximum de follicules en croissance. Par ailleurs, chez la ratte, de Reviers (1974) a montré que l'injection de doses croissantes de FSH, chez l'immature, provoque simultanément une augmentation du volume occupé par les follicules normaux et un accroissement encore plus important de celui occupé par les follicules atrésiques.

- Aux âges extrêmes étudiés, si le nombre total de follicules est équivalent, la distribution de leur taille diffère : à 11 semaines, quelques follicules ont des surfaces comprises entre $407000 \mu^{2}$ et $803000 \mu^{2}$. Ceux-ci croissent en nombre et en taille, dessinant une véritable deuxième population à 20 semaines dans laquelle le pourcentage d'atrésie est faible. II existe, dans cette poppulation, des follicules d'une surface de $571.000 \mu^{2}$ possédant des ovocytes reprenant la méiose. Cette observation donne de précieux renseignements sur la précocité de la réceptivité ovarienne envers les hormones gonadotropes et des indications sur les potentialités d'ovulation. En effet, le délai existant entre la formation des follicules à antrum et leur réponse aux gonadotrophines (problème de "l'insensibilité ovarienne ") est très variable selon les espèces : mois chez la femme, semaines chez la brebis, jours chez le cobaye, immédiat chez le hamster (Levasseur, 1979). Dès 11 semaines, l'étude histologique prouve que quelques femelles possèdent des follicules de tailles compatibles avec une reprise de méiose donc peu de temps après le début de formation des follicules à antrum ; et à 14 semaines, selon ce critère, presque toutes les femelles devraient ovuler. Dans cette optique, on peut citer les travaux de Hertz et Hisaw (1934) qui ont montré que des injections de HCG entre 12 et 14 semaines font ovuler un nombre élevé de lapines.

\section{Influence des conditions d'alimentation sur les différents caractères} étudiés. - Le rationnement à partir de 11 semaines à $75 \mathrm{p}$. 100 du régime ad libitum entraîne un retard de croissance corporelle d'environ 3 semaines ; aussi est-il logique d'observer une incidence de ce régime sur tous les caractères dépendant partiellement du poids.

La réceptivité sexuelle n'est que très temporairement amoindrie (contrairement aux observations de Van den Broeck et al., 1977), par contre, tous les phénomènes concernant l'ovulation sont touchés :

- Le déclenchement de la ponte ovulaire est retardé de 3 semaines également ; 
Short et al., (1968) observent sur la lapine adulte que l'effet du rationnement se manifeste, notamment par une décroissance de l'aptitude à ovuler. Van den Broeck et al. (1977) et Hammond (1965) constatent l'abaissement du taux de gestation.

- Le nombre d'ovules pondus est légèrement inférieur à 17 et 20 semaines par rapport au témoin. Short et al. (1968) et Hammond (1965) ont également constaté un moins grand nombre d'ovules pondus chez la lapine sous-alimentée. Ce phénomène peut être expliqué par un poids moyen des lapines rationnées inférieur et par l'état de la population folliculaire. En effet, au niveau de l'ovaire, la sous-alimentation pratiquée dans cette expérience ne modifie pas la population des petits follicules à antrum. Par contre, elle touche celle des follicules supérieurs à $407000 \mu^{2}$, à la fois moins nombreux, beaucoup moins gros et plus sensibles à l'atrésie. Au niveau de cette population, un retard sensible est enregistré à 17 semaines et est encore appréciable à 20 semaines.

\section{Conclusion.}

L'étude histologique de l'ovaire montre que de jeunes femelles de 11 et surtout 14 semaines possèdent des follicules à antrum dont la taille permet à l'ovocyte de reprendre la méïose. Or, l'aptitude à ovuler s'acquiert environ 3 semaines plus tard. Ces constatations suggèrent l'absence d'une décharge de LH suffisamment "importante " pour déclencher le phénomène. Différents mécanismes peuvent y contribuer. Foster et Ryan (1979) postulent l'existence d'une action " feedback » inhibitrice de l'œstradiol envers les sécrétions toniques de LH chez l'agnelle immature. II se peut également que la croissance pondérale ait une action partielle sur le fonctionnement hypophysaire. Ceci expliquerait que la lapine doive avoir un poids minimum pour acquérir l'aptitude à ovuler.

L'abaissement du poids, en liaison avec le rationnement, conduit à un ralentissement des fonctions endocriniennes (un état de « dietary hypophysectomy ", selon Lintern-Moore et Everitt (1978) qui retarde le démarrage de la puberté chez beaucoup d'espèces et plus précisément chez la lapine de l'ordre de 3 semaines. II est à souligner toutefois que le rationnement appliqué dans cette expérience (réduction de 25 p. 100 de la quantité ingérée ad libitum) ne conduit pas à une détérioration irréversible de l'ovaire (les petits follicules à antrum n'étant pas touchés) et explique qu'un " flushing " postérieur puisse restaurer rapidement les fonctions de reproduction (Van den Broeck et Lampo, 1972 ; Lintern-Moore et al., 1981).

Une étude de la cinétique des hormones gonadotropes s'avère un complément indispensable, à l'issue de ce travail, pour comprendre le sens de la " crise » de croissance folliculaire observée à 14 semaines et pour détecter l'apparition des premières décharges efficaces de LH. 


\section{Références}

ADAMS C. E., 1954. The experimental shortening of the generation interval. Proc. B.S.A.P., 97-108.

ASDELL, 1946. Patterns of mammalian reproduction. Comstock, Ithaca, New York, 44-192.

CALIGARIS L., ASTRADA J. J.. TALESNIK S., 1973. Development of the mechanisms involved in the facilitatory and inhibitory effects of ovarian steroids on the release of follicle stimulation hormone in the immature rat. J. Endocr., 58, 547-554.

CHERNEY D. D., DIDIO L. J. A., MOTTA P., 1975. The development of rabbit ovarian follicles following copulation. Fertil. Steril., 26, 257-270.

FOSTER D., RYAN K., 1979. Mechanisms governing onset of ovarian ciclicity at puberty in the lamb. Ann. Biol. anim. Bioch. Biophys., 19. 1369-1380.

GREGORY P. W., 1932. Potential and actual fecundity of some breeds of rabbit. J. exp. Zool., 62. 271-285.

HAMMOND J., 1965. The effects of high and low planes of nutrition on reproduction in rabbits. N. Z, J. agric. Res., 8, 708-717.

HERTZ R., HISAW F., 1934. Effects of follicle-stimulating and luteinizing pituitary extracts on the ovaries of the infantile and juvenile rabbit. Am. J. Physiol., 108, 1-13.

HULOT F., MATHERON G., 1981. Effets du génotype, de l'âge et de la saison sur les composantes de la reproduction chez la lapine. Ann. Génét. Sél. anim., 13, 131-150.

KENNEDY J. P., WORTHINGTON C. A., COLE E. R., 1974. The postnatal development of the ovary and uterus of the Merino lamb. J. Reprod. Fert., 36, 275-282.

LEVASSEUR M. C., 1979. Thoughts on puberty. The gonads, Ann. Biol. anim. Bioch. Biophys., 19, 321-335.

LINTERN-MOORE S., EVERITT A. V., 1978. The effect of restricted food intake on the size and composition of the ovarian follicle population in the Wistar rat. Biol. Reprod., 19, 688-691.

LINTERN-MOORE S., EVERITT A. V., MARIANA J. C., MAULÉON P., 1981. The effect of restricted food intake and refeeding on the ovarian follicle population of the pre-puberal Wistar rat. Reprod. Nutr. Dévelop., 21, 611-620.

MARIANA J. C., 1972. Classification des follicules ovariens. Ann. Biol. anim. Bioch. Biophys., 12, 377-382.

MAULÉON P., 1961. Déroulement de l'ovogenèse comparé chez différents mammifères domestiques. Proc. 4th int. Congr. anim. Reprod. The Hague, 2, 348-354.

MAULÉON P., MARIANA J. C., 1977. Oogenesis and folliculogenesis, 175-201. In COLE H. H., CUPPS P. T., Reproduction in domestic animals. Acad. Press.

OJEDA S. R., RAMIREZ V. D., 1972. Plasma level of LH and FSH in maturing rats : response to hemigonadectomy. Endocrinology, 90, 466-472.

PEDERSEN T., 1969. Follicle growth in the immature mouse ovary. Acta endocr., 62, 117-132.

PEDERSEN T., 1970. Determination of follicle growth rate in the ovary of the immature mouse. J. Reprod. Fert., 17, 555-557.

PETERS H., 1969. The development of the mouse ovary from birth to maturity. Acta endocr., 62, 98-116.

REVIERS M. M. de, 1974. Etude quantitative de l'action des hormones gonadotropes hypophysaires sur la population folliculaire de l'ovaire de ratte immature. Signification biologique du dosage de l'hormone folliculostimulante par le test de Steelman et Pohley. Thèse Doct. ès Sci. Univ., Tours, $67 \mathrm{pp}$.

SHORT R. E., PETERS J. B., CASIDA L. E., 1968. Effect of two levels of feeding on ovarian activity, embryo survival and ovarian compensatory hypertrophy in the rabbit. J. anim. Sci. 27, 701-704.

THIBAULT C., GERARD M., MENEZO Y., 1976. Nuclear and cytoplasmic aspects of mammalian oocyte maturation in vitro in relation to follicle size and fertilization sperm action. Prog. Reprod. Biol., 1, 233-240, Karger, Basel.

VAN den BROECK L., LAMPO Ph., 1977. Influence de trois niveaux d'alimentation de lapines futures reproductrices sur l'ardeur sexuelle et la fertilité à 4 mois. Ann. Zootech., 26, 565-574.

VENGE O., 1950. Studies of the maternal influence on the birth weight in rabbits. Acta. zool., 31, 1-48.

WIDDOWSON E. M., MAVOR W. O., Mc CANCE R. A., 1964. The effect of undernutrition and rehabilitation on the development of reproductive organs: rats. J. Endocrinol., 29, 119-126.

YASCHINE T., MENA F., BEYER C., 1967. Gonadal hormones and mounting behavior in the female rabbit. Am. J. Physiol., 213, 867-872. 